\title{
GMR
}

\section{Development, characterization, and annotation of potential simple sequence repeats by transcriptome sequencing in pears (Pyrus pyrifolia Nakai)}

\author{
H. Zhou, B.H. Cai, Z.Q. Lü, Z.H. Gao and Y.S. Qiao \\ Laboratory of Fruit Tree Biotechnology, College of Horticulture, \\ Nanjing Agricultural University, Nanjing, China \\ Corresponding author: Y.S. Qiao \\ E-mail: qiaoyushan@njau.edu.cn \\ Genet. Mol. Res. 15 (3): gmr.15038683 \\ Received March 31, 2016 \\ Accepted July 15, 2016 \\ Published September 23, 2016 \\ DOI http://dx.doi.org/10.4238/gmr.15038683
}

Copyright (C) 2016 The Authors. This is an open-access article distributed under the terms of the Creative Commons Attribution ShareAlike (CC BY-SA) 4.0 License.

\begin{abstract}
Simple sequence repeats (SSRs), one of the most powerful molecular markers, can be used for DNA fingerprinting, variety identification, genetic mapping, and marker-assisted selection. Using the pear's (Pyrus pyrifolia Nakai) 75,764 unigenes (55,676,271 bp) obtained by deep transcriptome sequencing, a total of 10,622 novel SSRs were identified in 9154 unigenes, accounting for $14.02 \%$ of all unigenes. The average length and distribution of these SSRs was about $16 \mathrm{bp}$ and $5.24 \mathrm{~kb}$, respectively. Dinucleotide repeat motifs were the main type, with a frequency of $55.87 \%$, followed by trinucleotides $(24.45 \%)$. There were 159 kinds of repeat motifs existing in the pear transcriptome. $\mathrm{AG} / \mathrm{CT}$ was the most frequent motif, accounting for $49.64 \%$. All 9154 SSR-containing unigenes were functionally annotated using $\mathrm{Nr}$ (NCBI non-redundant protein database), $\mathrm{Nt}$ (NCBI non-redundant nucleotide
\end{abstract}


database), and the Swiss-Prot database, and were classified further by Gene Ontology and Clusters of Orthologous Groups. In addition, a total of 4300 primer pairs were designed from all SSR loci obtained. Of these, 40 primers were randomly selected for PCR amplification and polyacrylamide gel (PAGE) analysis. Among the 40 primer pairs, 31 were successfully separated via PAGE. These findings also confirm that mining SSRs using next-generating sequencing technologies is a fast, effective, and reliable approach.

Key words: Simple sequence repeats; Pyrus pyrifolia Nakai; Transcriptome; Development of primers; Functional annotation

\section{INTRODUCTION}

Pears (Pyrus spp) belong to the genus Pyrus within the family Rosaceae and are the third most important temperate fruits after grapes and apples (Potter et al., 2007; Wu et al., 2013). Pyrus is considered to be typically self-incompatible. Its phylogenetic relationships are complicated and difficult to establish because of high heterozygosity and poor morphological diversity. Hence, the development of highly polymorphic molecular markers is essential for resolving these problems in breeding programs and other genetic research on pears.

SSRs, or microsatellites, are repetitive DNA sequences consisting of motif repeats (1-6 bp) with conservative flanking sequences. SSR markers are among the most valuable and efficient molecular markers because of their good genome coverage, genetic co-dominant inheritance, polymorphism, and reproducibility (Tautz and Renz, 1984; Shen et al., 2015). In addition, since the use of SSRs is based on PCR amplification, the technique is simple and only a small amount of DNA is required. SSRs can be classified into two categories according to their source: genomic SSRs (DNA-derived SSRs) and EST-SSRs/genic SSRs (expressed sequence tag-SSRs). DNA-derived SSRs are derived from SSR-enriched genomic libraries or random genomic sequences. In contrast, EST-SSRs are usually derived from the transcriptome or EST sequences in public databases. Compared with DNA-derived SSRs, EST-SSRs are considered more valuable molecular markers, to a certain extent, because of their functionality, co-specific transferability, and polymorphisms, especially in some specific genetic studies.

Traditional methods for the development of DNA-derived SSRs are expensive, timeconsuming, and laborious. SSRs developed from publicly available genetic/genomic information of the species of interest to us, is the most effective method. However, the dearth of available sequences is a serious obstacle in the application of this method. Mining EST-SSR markers based on transcriptome information generated by large-scale sequencing (such as Roche/454, Illumina/Solexa, and ABI/Solid platforms) is an effective strategy to overcome this problem.

The genome of Pyrus bretschneideri Rehd. 'Dangshansu' has been previously sequenced (Wu et al., 2013) and used to develop genomic SSRs (Fan et al., 2013). Some developments in pear EST-SSRs have also been recently reported (Yue et al., 2014; Zhang et al., 2014). However, only a few EST-SSRs, which were developed from pear transcriptome sequencing, have been reported. The number of publicly available SSRs is still too low for use in breeding selection, phylogenetic relationship analysis, and other genetic studies in pears. Hence, developing more EST-SSRs from pears would be desirable.

To our knowledge, we here report the first case of functional annotation of pear

Genetics and Molecular Research 15 (3): gmr.15038683 
genic SSRs-containing sequences, and this is also one of the few studies where EST-SSRs were developed from the pear transcriptome. The frequency, type, distribution, and other features of these EST-SSRs are also reported in our paper. The thousands of EST-SSRs reported in our paper can be used to enrich molecular markers and accelerate genetic research in pears, and they can also be used in genetic studies of other members of Rosaceae, because of its high co-specific transferability and polymorphism, especially in those species with limited available sequences.

\section{MATERIAL AND METHODS}

\section{Plant materials and data sources}

The transcriptome data used in this study were from high-throughput sequencing of Pyrus pyrifolia Nakai 'Huanghua' peels. P. pyrifolia 'Huanghua' was grown in the Lishui Orchard (Nanjing, China). Fruits were collected at the following developmental times: 6, 7, 8, and 9 weeks after flowering. Total RNA was isolated by the modified CTAB method (Wang et al., 2010a). RNA quality was confirmed by analyzing samples with a 2100 Bioanalyzer (Agilent Technologies, Palo Alto, CA, USA), with a minimum RIN value of 8.2. In total, 20 $\mu \mathrm{g}$ RNA was equally pooled from the four samples for cDNA library preparation. Using a Solexa/Illumina HiSeq ${ }^{\mathrm{TM}} 2000$ RNA-seq platform, 53,856,740 high-quality reads (more than $4.8 \mathrm{G}$ ) were obtained, trimmed, and assembled into 75,764 unigenes.

\section{DNA extraction}

Total genomic DNA was extracted from fresh, young P. pyrifolia Nakai 'Huanghua' leaves by the modified CTAB method (Pan et al., 2006). DNA quality and quantity was confirmed with 1\% agarose E-Gels (Invitrogen, Shanghai, China) and an Eppendorf BioPhotometer (Eppendorf, Hamburg, Germany), respectively. The DNA was diluted in sterilized $\mathrm{ddH}_{2} \mathrm{O}$ to a concentration of $100 \mathrm{ng} / \mu \mathrm{L}$ and stored at $-20^{\circ} \mathrm{C}$ for PCR analysis.

\section{SSR locus search}

The MIcroSAtellite software (MISA, http://pgrc.ipk-gatersleben.de/misa/), which is based on the Perl language, was performed to detect SSR loci using all the 75,764 unigenes as references. SSRs were defined as mononucleotide, dinucleotide, trinucleotide, tetranucleotide, pentanucleotide, and hexanucleotide, with a minimum of $12,6,5,5,4$, and 4 repetitions for all motifs, respectively. The SSRs detected were classified into two groups: perfect SSRs and compound SSRs. The maximum number of bases interrupting perfect SSRs in a compound SSR was 100.

\section{SSR primer design}

Primer3 v2.3.4 was used to design primers in the flanking regions (when greater than $150 \mathrm{bp}$ ) of SSR loci on unigenes. The design process was based on the following criteria: 1) The length of primers was between 18-28 bp, with an optimum size of $23 \mathrm{bp} ; 2$ ) an annealing temperature between $55^{\circ}-65^{\circ} \mathrm{C}$, with a maximum discrepancy within $2^{\circ} \mathrm{C}$ among forward/ reverse primers; and 3) PCR product size ranging from 80 to $160 \mathrm{bp}$. Other parameters were set at the default setting of the software.

Genetics and Molecular Research 15 (3): gmr.15038683 
Five pairs of primers were designed for each SSR locus. Then, primers were screened as follows: 1) no SSRs were present within the primers; 2) the primers were aligned to unigene sequences, with the 5'-site containing no more than 3 mismatches; 3 ) removal of the primers that were aligned to more than one unigene; and 4) SSRs on the product sequences were located with SSR_finder (http://www.fresnostate.edu/ssrfinder/), and the products retained from SSR_finder's results were the same as those of MISA.

\section{Verification of SSR primers}

Forty SSR primer pairs were randomly selected from the 4300 primers for PCR amplification. Each PCR was carried out in a total final volume of $25 \mu \mathrm{L}$, containing: $1 \mu \mathrm{L} 100$ ng/ $\mu \mathrm{L}$ genomic DNA, $2.5 \mu \mathrm{L}$ 10X PCR Buffer, $2.0 \mu \mathrm{L} 2.5 \mathrm{mM}$ dNTP, $1.5 \mu \mathrm{L} \mathrm{MgCl}_{2}(25 \mathrm{mM})$, $2.0 \mu \mathrm{L}$ forward and reverse primer $(10 \mathrm{pM})$, and $0.2 \mu \mathrm{L} 5 \mathrm{U} / \mu \mathrm{L}$ Taq DNA polymerase (Takara Biotechnology, Dalian, China). All SSR amplifications were performed under the following conditions: initial denaturation temperature at $94^{\circ} \mathrm{C}$ for $4 \mathrm{~min}$, followed by 8 cycles at $94^{\circ} \mathrm{C}$ for $30 \mathrm{~s}, 59^{\circ} \mathrm{C}$ for $30 \mathrm{~s}$, and $72^{\circ} \mathrm{C}$ for $30 \mathrm{~s}$, where the annealing temperature was reduced by $0.5^{\circ} \mathrm{C}$ per cycle. This was then followed by 30 cycles at $94^{\circ} \mathrm{C}$ for $30 \mathrm{~s}, 56^{\circ} \mathrm{C}$ for $30 \mathrm{~s}, 72^{\circ} \mathrm{C}$ for $30 \mathrm{~s}$, and a final extension for $10 \mathrm{~min}$ at $72^{\circ} \mathrm{C}$. The amplification products and the same amount of ladder markers (20-bp DNA Ladder Marker, D512A) were loaded onto an 8\% nondenaturing PAGE. The gel was silver-stained and photographed. Taq DNA polymerase, dNTP, and DNA Ladder Marker were purchased from Takara Biotechnology (Dalian).

\section{Functional annotation for SSR-containing unigenes}

Functional annotation was also performed. All SSR-containing unigenes were searched using BLAST against the Nr, Nt, Swiss-prot, and COG, with a cut-off e-value of $10^{-5}$. These SSR-containing unigene sequences were first aligned with BLASTx to the protein databases Nr and Swiss-prot, and aligned by BLASTn to the nucleotide database Nt (e-value of $10^{-5}$ ). Functional classification by GO was analyzed with Blast2GO (Conesa et al., 2005). After acquiring a GO annotation for every unigene, the WEGO software was used to perform GO functional classification (Ye et al., 2006). The unigenes were also aligned to the COG database to predict and classify possible functions using the BLAST software (e-value of $10^{-5}$ ).

\section{RESULTS}

\section{Frequency, distribution, and other features of SSRs in the pear transcriptome}

Of the 75,764 unigenes $(55,676,271 \mathrm{bp})$ derived from the 'Huanghua' pear transcriptome, a total of 10,622 SSRs containing 1-6-bp repeat motifs were identified in 9154 unigenes, of which 1261 unigenes contained more than one SSR (Table 1). Six hundred and forty-four compound SSRs were also detected. In the SSR statistics and primer pairs designed, we separated a compound SSR into several perfect SSRs.

Mononucleotide and dinucleotide were the major types, accounting for $80.32 \%$ of all SSR motifs (Table 2). The most abundant motif was dinucleotide $(5935,55.87 \%)$, followed by trinucleotide $(2597,24.45 \%)$ and mononucleotide $(1539,14.49 \%)$. The total number of tetranucleotides, pentanucleotides, and hexanucleotides was only 551 (5.19\%). The frequency

Genetics and Molecular Research 15 (3): gmr.15038683 
and average distribution distance of these 10,622 SSRs was about $14.02 \%$ and $5.24 \mathrm{~kb}$, respectively. In our study, the total lengths of all SSRs were up to 173,342 bp, with an average length of $16 \mathrm{bp}$, including mononucleotides $(23,819 ; 15 \mathrm{bp})$, dinucleotides $(93,506 ; 16 \mathrm{bp})$, trinucleotides $(43,746,17 \mathrm{bp})$, tetranucleotides $(3016 ; 21 \mathrm{bp})$, pentanucleotides $(4455 ; 21 \mathrm{bp})$, and hexanucleotides $(4800 ; 25 \mathrm{bp})$. The incidence of different repeats and frequencies of each motif was also evaluated (Table 2). The most common class was $\mathrm{N}=6(21.34 \%)$, which contained mostly dinucleotide repeats, followed by $\mathrm{N}=5(15.96 \%)$ and $\mathrm{N}=7(13.67 \%)$.

\begin{tabular}{l|c} 
Table 1. SSR search results using MISA. & Number \\
\hline Item & 75,764 \\
\hline Total number of unigenes examined & $55,676,271$ \\
\hline Total size of examined sequences (bp) & 10,622 \\
\hline Total number of identified SSRs & 9154 \\
\hline Number of SSR-containing unigenes & 1261 \\
\hline Number of unigenes containing more than one SSR & 644 \\
\hline Number of compound SSRs & \\
\hline
\end{tabular}

Table 2. Frequency of different SSR repeat motifs.

\begin{tabular}{|c|c|c|c|c|c|c|c|c|}
\hline \multirow[t]{2}{*}{ Repeat number } & \multicolumn{6}{|c|}{ SSR motif } & \multirow[t]{2}{*}{ Total } & \multirow[t]{2}{*}{$\%$} \\
\hline & Mono- & Di- & Tri- & Tetra- & Penta- & Hexa- & & \\
\hline 4 & - & - & - & - & 192 & 165 & 357 & 3.36 \\
\hline 5 & - & - & 1539 & 123 & 21 & 12 & 1695 & 15.96 \\
\hline 6 & - & 1571 & 664 & 22 & 3 & 7 & 2267 & 21.34 \\
\hline 7 & - & 1117 & 331 & 1 & 0 & 3 & 1452 & 13.67 \\
\hline 8 & - & 1065 & 43 & 0 & 0 & 1 & 1109 & 10.44 \\
\hline 9 & - & 1116 & 0 & 0 & 0 & 1 & 1117 & 10.52 \\
\hline 10 & - & 857 & 3 & 0 & 0 & 0 & 860 & 8.10 \\
\hline 11 & - & 186 & 2 & 0 & 0 & 0 & 188 & 1.77 \\
\hline 12 & 353 & 19 & 10 & 0 & 0 & 0 & 382 & 3.60 \\
\hline 13 & 271 & 0 & 0 & 0 & 0 & 0 & 271 & 2.55 \\
\hline 14 & 178 & 0 & 5 & 0 & 0 & 0 & 183 & 1.72 \\
\hline$>14$ & 737 & 4 & 0 & 0 & 0 & 0 & 741 & 6.98 \\
\hline Total & 1539 & 5935 & 2597 & 146 & 216 & 189 & 10,622 & 100 \\
\hline$\%$ & 14.49 & 55.87 & 24.45 & 1.37 & 2.03 & 1.78 & 100 & \\
\hline
\end{tabular}

Within the detected SSRs, 159 kinds of repeat motifs were identified, of which mono-, di-, tri-, tetra-, penta-, and hexanucleotide repeat motifs had 2, 4, 10, 23, 45, and 75 types, respectively (Table 3). AG/CT was the most frequent motif, accounting for $49.64 \%$ of all SSRs, followed by A/T (1515, 14.26\%), AAG/CTT (662, 6.23\%), AGG/CCT (544, 5.12\%), AGC/CTG (444, 4.18\%), AC/GT (391, 3.68\%), ACC/GGT (335, 3.15\%), AT/AT (260, 2.45\%), and ATC/ATG (204, 1.92\%). The frequency of the remaining 150 types of motifs accounted for $9.36 \%$ (Table 4 ).

$\mathrm{AG} / \mathrm{CT}$ comprised $88.85 \%$ of all dinucleotide motifs and was the most common type among all SSR repeats (Figure 1A). The predominant trinucleotide motifs were AAG/CTT (25.49\%) and AGG/CCT (20.95\%) (Figure 1B).

\section{Development and verification of SSR primers}

All 10,622 SSRs were screened, and the SSRs wherein the lengths of both flanking regions on the unigene greater than $150 \mathrm{bp}$ were kept to design primers. Finally, 2526 (23.78\%) SSRs were retained, and 12,630 primer pairs were designed.

Genetics and Molecular Research 15 (3): gmr.15038683 
Table 3. Type and number of repeat motifs.

\begin{tabular}{|c|c|c|}
\hline Repeat type & Repeat motif & Number \\
\hline Mono- & $\mathrm{A} / \mathrm{T}, \mathrm{C} / \mathrm{G}$ & 2 \\
\hline Di- & $\mathrm{AC} / \mathrm{GT}, \mathrm{AG} / \mathrm{CT}, \mathrm{AT} / \mathrm{AT}, \mathrm{CG} / \mathrm{CG}$ & 4 \\
\hline Tri- & $\begin{array}{l}\text { AAC/GTT, AAG/CTT, AAT/ATT, ACC/GGT, ACG/CGT, ACT/AGT, AGC/CTG, AGG/CCT, } \\
\text { ATC/ATG, CCG/CGG }\end{array}$ & 10 \\
\hline Tetra- & $\begin{array}{l}\text { AAAC/GTTT, AAAG/CTTT, AAAT/ATTT, AACC/GGTT, AACT/AGTT, AAGC/CTTG, } \\
\text { AAGG/CCTT, AATC/ATTG, AATG/ATTC, AATT/AATT, ACAG/CTGT, ACAT/ATGT, } \\
\text { ACCG/CGGT, ACGT/ACGT, ACTC/AGTG, ACTG/AGTC, AGAT/ATCT, AGCC/CTGG, } \\
\text { AGCG/CGCT, AGGC/CCTG, AGGG/CCCT, ATCC/ATGG, ATCG/ATCG }\end{array}$ & 23 \\
\hline Penta- & $\begin{array}{l}\text { AAAAC/GTTTT, AAAAG/CTTTT, AAAAT/ATTTT, AAACC/GGTTT, AAACG/CGTTT, } \\
\text { AAAGC/CTTTG, AAAGG/CCTTT, AAATC/ATTTG, AAATT/AATTT, AACAC/GTGTT, } \\
\text { AACAG/CTGTT, AACCC/GGGTT, AACCG/CGGTT, AACGG/CCGTT, AAGAG/CTCTT, } \\
\text { AAGAT/ATCTT, AAGGG/CCCTT, AATAC/ATTGT, AATCC/ATTGG, AATCG/ATTCG, } \\
\text { AATCT/AGATT, AATGG/ATTCC, AATTC/AATTG, ACACG/CGTGT, ACAGC/CTGTG, } \\
\text { ACAGT/ACTGT, ACCAG/CTGGT, ACCAT/ATGGT, ACCTC/AGGTG, ACGCC/CGTGG, } \\
\text { ACGGG/CCCGT, ACTAG/AGTCT, ACTCC/AGTGG, ACTCG/AGTCG, ACTGG/AGTCC, } \\
\text { AGAGC/CTCTG, AGAGG/CCTCT, AGATC/ATCTG, AGATG/ATCTC, AGCTC/AGCTG, } \\
\text { AGGCC/CCTGG, AGGGG/CCCCT, ATCCC/ATGGG, ATCCG/ATCGG, ATCGC/ATGCG }\end{array}$ & 45 \\
\hline Hexa- & $\begin{array}{l}\text { AAAAAC/GTTTTT, AAAAAG/CTTTTT, AAAAAT/ATTTTT, AAAACC/GGTTTT, } \\
\text { AAAAGC/CTTTTG, AAAATT/AATTTT, AAACCG/CGGTTT, AAACGG/CCGTTT, } \\
\text { AAAGAG/CTCTTT, AAAGCC/CTTTGG, AAAGGG/CCCTTT, AAATAC/ATTTGT, } \\
\text { AAATCG/ATTTCG, AAATGG/ATTTCC, AAATTC/AATTTG, AACACC/GGTGTT, } \\
\text { AACCAC/GGTTGT, AACCAG/CTGGT, AACCCC/GGGGT, AACCCG/CGGGT, } \\
\text { AACCCT/AGGGTT, AACCGC/CGGTTG, AACTCC/AGTTGG, AACTTG/AAGTTC, } \\
\text { AAGACG/CGTCTT, AAGAGG/CCTCTT, AAGATG/ATCTTC, AAGCAC/CTTGTG, } \\
\text { AAGCAG/CTGCTT, AAGGAG/CCTTCT, AAGGGG/CCCCTT, AAGGTC/ACCTTG, } \\
\text { AAGTCC/ACTTGG, AATCCC/ATTGGG, AATCTC/AGATTG, AATGGG/ATTCCC, } \\
\text { AATTCC/AATTGG, ACACAG/CTGTGT, ACACCC/GGGTGT, ACACCT/AGGTGT, } \\
\text { ACAGAG/CTCTGT, ACAGGC/CCTGTG, ACAGGG/CCCTGT, ACATCC/ATGTGG, } \\
\text { ACATGG/ATGTCC, ACCACG/CGTGGT, ACCAGC/CTGGTG, ACCATC/ATGGTG, } \\
\text { ACCATG/ATGGTC, ACCGAG/CGGTCT, ACCGCC/CGGTGG, ACCGTC/ACGGTG, } \\
\text { ACCTCC/AGGTGG, ACGAGG/CCTCGT, ACGATC/ATCGTG, ACGGAG/CCGTCT, } \\
\text { ACGGCG/CCGTCG, ACGGGC/CCCGTG, ACTAGC/AGTGCT, ACTCCC/AGTGGG, } \\
\text { ACTCTC/AGAGTG, ACTGCG/AGTCGC, ACTGCT/AGCAGT, AGAGCC/CTCTGG, } \\
\text { AGAGGC/CCTCTG, AGAGGG/CCCTCT, AGATCT/AGATCT, AGCAGG/CCTGCT, } \\
\text { AGCCTC/AGGCTG, AGGATG/ATCCTC, AGGCGG/CCGCCT, AGGGAT/ATCCCT, } \\
\text { AGGGGG/CCCCCT, ATCCCC/ATGGGG, ATCGCC/ATGGCG }\end{array}$ & 75 \\
\hline
\end{tabular}

Table 4. Frequency distribution of different SSR motifs.

\begin{tabular}{l|c|c|l|c|c}
\hline SSR motif & Frequency of SSR motif & $\%$ & SSR motif & Frequency of SSR motif & $\%$ \\
\hline A/T & 1515 & 14.26 & AGC/CTG & 444 & 4.18 \\
\hline C/G & 24 & 0.23 & ACC/GGT & 335 & 3.15 \\
\hline AG/CT & 5273 & 49.64 & ATC/ATG & 204 & 1.92 \\
\hline AC/GT & 391 & 3.68 & CCG/CGG & 114 & 1.07 \\
\hline AT/TA & 260 & 2.45 & AAC/GTT & 109 & 1.03 \\
\hline CG/GC & 11 & 6.23 & AAT/ATT & 46 & 0.43 \\
\hline AAG/CTT & 662 & 5.12 & ACT/AGT & 32 & 0.30 \\
\hline AGG/CCT & 544 & 5.19 & & & \\
\hline Others & 551 &
\end{tabular}

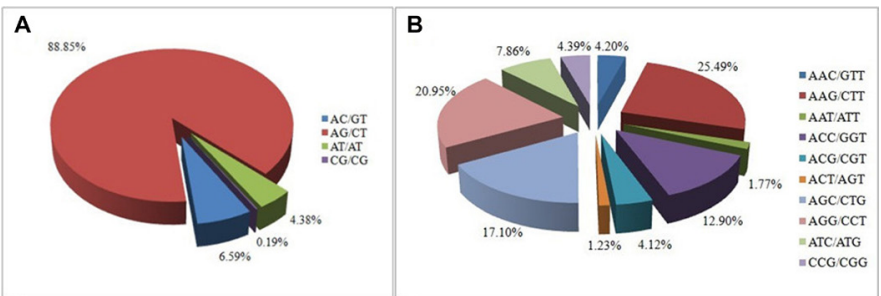

Figure 1. Percentage of different dinucleotide and trinucleotide motifs.

Genetics and Molecular Research 15 (3): gmr.15038683 
These primers were filtered by the previously mentioned criteria, and 4300 primers were obtained, including $30(0.70 \%)$ for mononucleotide repeats, $1388(32.28 \%)$ for dinucleotide repeats, $2623(61.00 \%)$ for trinucleotide repeats, $49(1.14 \%)$ for tetranucleotide repeats, $97(2.26 \%)$ for pentanucleotide repeats, and $113(2.63 \%)$ for hexanucleotide repeats (Table S1). AT/TA did not exist among these 4300 primer pairs. AT/AT is not usually used to develop markers because of its self-complementary nature, which can lead to palindrome formation (Wang et al., 2011). A total of 40 primer pairs were randomly selected for PCR amplification and PAGE analysis (Table 5).

Among the 40 primer pairs, 31 were successfully amplified by PCR and PAGE (Figure 2). The remaining 9 primers failed to generate PCR products at various annealing temperatures and $\mathrm{Mg}^{2+}$ concentrations. Among the 31 successfully amplified primer pairs, 29 met the expected size and 2 were smaller products than expected.

\section{Functional annotation for SSR-containing unigene sequences}

All 9154 SSR-containing unigenes were functionally annotated by searches against the Nr, Nt, and Swiss-prot databases. All SSR-containing unigenes were searched using BLASTx against the $\mathrm{Nr}$ database, using a cut-off e-value of $10^{-5}$, and 6485 unigenes $(70.84 \%$ of all SSR-containing unigenes) were annotated within the database. These unigenes were also aligned by BLASTx to the protein database Swiss-prot, and BLASTn to the Nt database, using a cut-off e-value of $10^{-5}$. From the Swiss-prot and Nt databases, a total of $4571(49.93 \%)$ and $6870(75.05 \%)$ unigenes were annotated, respectively.

These unigenes were classified further using GO terms and COG categories. GO has three major categories (ontologies): molecular function, cellular component, and biological process. The basic unit of GO is the GO-term. Every GO-term belongs to a type of ontology. With $\mathrm{Nr}$ annotation, we used Blast2GO to generate GO annotations of the unigenes. After acquiring a GO annotation for every unigene, we used WEGO to perform a GO functional classification for all SSR-containing unigenes. Based on sequence homology, 5339 (58.32\%) unigenes were assigned to 35,934 GO-term annotations. These 35,934 GO-terms were summarized into the three major categories. Of them, biological process $(18,679,51.98 \%)$ comprised the majority of the $\mathrm{GO}$ annotations, followed by cellular component $(9151,25.47 \%)$ and molecular function $(8104,22.55 \%)$.

COG is a database where orthologous gene products are classified. Every protein in COG was assumed to have evolved from an ancestral protein, and the whole database was built on coding proteins with complete genomes as well as system evolution relationships of bacteria, algae, and eukaryotic organisms. In our study, unigenes were aligned to the COG database to predict and classify their possible functions. In total, 2770 (30.26\%) unigenes were assigned to the COG classification, with 3924 COG functional annotations (Table 6). Among 25 COG categories, the cluster for 'General function prediction only' $(759,27.40 \%)$ represented the largest group, followed by 'Transcription' $(513,18.52 \%)$, 'Replication, recombination and repair' $(338,12.20 \%)$, and 'Signal transduction mechanisms' $(328,11.84 \%)$. The following clusters: 'Cell motility', 'Extracellular structures', and 'Nuclear structure' (no unigenes were assigned to this group) were the smallest groups.

Genetics and Molecular Research 15 (3): gmr.15038683 
Table 5. Forty validated primer pairs used in the study.

\begin{tabular}{|c|c|c|c|c|c|c|}
\hline Code & Primer & $\begin{array}{l}\text { SSR } \\
\text { motif }\end{array}$ & $\begin{array}{c}\text { Number } \\
\text { of repeats }\end{array}$ & Primer sequence $\left(5^{\prime} \rightarrow 3^{\prime}\right)$ & $\begin{array}{l}\text { Except } \\
\text { size/bp }\end{array}$ & Product \\
\hline \multirow[t]{2}{*}{1} & \multirow[t]{2}{*}{ NAUPP_0014 } & \multirow[t]{2}{*}{ GAT } & \multirow[t]{2}{*}{7} & GTTCTCTTCTGTTTGAGAAGTGCT & \multirow[t]{2}{*}{113} & \multirow[t]{2}{*}{ ES } \\
\hline & & & & CTCACTCAGGATCTCAGTCAGGT & & \\
\hline \multirow[t]{2}{*}{2} & \multirow[t]{2}{*}{ NAUPP_0034 } & \multirow[t]{2}{*}{$\mathrm{AC}$} & \multirow[t]{2}{*}{11} & ACACTGTTTTGGTGTTATGCTTG & \multirow[t]{2}{*}{132} & \multirow[t]{2}{*}{ NA } \\
\hline & & & & CTCCAAAGCTCTCСТCTTTCTTT & & \\
\hline \multirow[t]{2}{*}{3} & \multirow[t]{2}{*}{ NAUPP_0041 } & \multirow[t]{2}{*}{ AG } & \multirow[t]{2}{*}{9} & GTTTTCGAGAACAAGACGAAGAC & 143 & ES \\
\hline & & & & TGCCCСТTCTTAATTCCTTCTTA & & \\
\hline 4 & NAUPP_0051 & GGT & 6 & GTCCAAACAGGAAGATGTGAAAC & 154 & ES \\
\hline & & & & ATCTGAAACTCCATCACCCCTAT & & \\
\hline 5 & NAUPP_0066 & GGA & 6 & ATCGAAATTAGGTTTGTGGGTTT & 158 & NA \\
\hline & & & & CATCTTCTTCCCTATCCCCATAC & & \\
\hline 6 & NAUPP_0181 & TGAGGA & 6 & GTAGTAGTAAGTGGGCTGGGAGG & 143 & ES \\
\hline & & & & CTTCTCCGCCTTCATCTCC & & \\
\hline 7 & NAUPP_0211 & GAGC & 6 & TACATGGACATTCTGAGAGACCC & 130 & NA \\
\hline & & & & AAATCAAAACCCCGAATTAAGAG & & \\
\hline 8 & NAUPP_0298 & GAC & 6 & ATGACAGCGTAGACGAAGAGGA & 136 & ES \\
\hline & & & & GACTGTACGACCTCCTGAACATC & & \\
\hline 9 & NAUPP_0490 & CGC & 7 & TATGGTACTTTTTCTTCCTCGGC & 146 & NA \\
\hline & & & & TTGCTCATATCTTCCTGTTGGTT & & \\
\hline 10 & NAUPP_0613 & GAG & 5 & TTAGGACAGGACTAACTGGAGCA & 151 & ES \\
\hline & & & & CAGAATTCCCATAAATCTGCATC & & \\
\hline 11 & NAUPP_0905 & AGTAC & 5 & ACTCGATGAAATGGAAGTTCGT & 134 & ES \\
\hline & & & & ACGCGCAATTATTGACCTTATAC & & \\
\hline 12 & NAUPP_1129 & GGAAA & 4 & TTTGTTTTTGGATGGAAAGATTG & 81 & ES \\
\hline & & & & CTTTCAAGAATCCCATTCCTCTC & & \\
\hline 13 & NAUPP_1148 & TA & 7 & AATTAATAAGCCCAGCCCTACAT & 131 & ES \\
\hline & & & & CCCACCTCTCAATGGTAAGCTA & & \\
\hline 14 & NAUPP_1298 & $\mathrm{AG}$ & 7 & CCTTATAAAGTTGGTGATGTGGG & 130 & ES \\
\hline & & & & CGAGGAACTCCATCCTCTACATA & & \\
\hline 15 & NAUPP_1552 & CTT & 7 & CGAGAGGTCATAGTCATCCATGT & 150 & ES \\
\hline & & & & GATTCAGTCGATTGTTGAGGAAG & & \\
\hline 16 & NAUPP_1639 & CAAA & 5 & AGAGAGTAGAAAACCGAAGCCTG & 117 & ES \\
\hline & & & & TCGGTAACTTTGCAATCTTTCAT & & \\
\hline 17 & NAUPP_1659 & $\mathrm{T}$ & 14 & AACCCACTAAGCAAAAGGTGAAT & 149 & ES \\
\hline & & & & GGACTCAAATTCCCATCATAACA & & \\
\hline 18 & NAUPP_1731 & CTT & 5 & GCCCAAAAGACCTCCTTCTC & 137 & ES \\
\hline & & & & CTGAACAGCACCAATGTTTTTG & & \\
\hline 19 & NAUPP_1846 & TTTTC & 4 & AAACTGATGTCAGGGCTCAAGTA & 128 & ES \\
\hline & & & & TGAAACCAACATGTCACACCTTA & & \\
\hline 20 & NAUPP_2033 & AT & 8 & CATCTCCATTTCCTGTGATTTTC & 133 & ES \\
\hline & & & & TTTGAGGGTGATGATGTAAAAGC & & \\
\hline 21 & NAUPP_2098 & CT & 6 & AGTTTTTGGATTTGCTGGTTGT & 150 & ES \\
\hline & & & & CAACAAATCCCTACTTTGAGAAATC & & \\
\hline 22 & NAUPP_2184 & AAGCAG & 4 & AGAAGGAAAGATGGGGTTTGAT & 138 & NA \\
\hline & & & & TCCTCTTTCTTGAGTTTGTCAGC & & \\
\hline 23 & NAUPP_2292 & CT & 8 & GAGTCCCAGACTGTCTCTCTGTC & 117 & ES \\
\hline & & & & ATGATGGGATCGTTATCAGTGTC & & \\
\hline 24 & NAUPP_2390 & $\mathrm{AG}$ & 6 & GTTTTCTCACCATAACTGCCTGT & 155 & ES \\
\hline & & & & TTGCATTTTAATTTTGGGTTTTG & & \\
\hline 25 & NAUPP_2446 & $\mathrm{TC}$ & 8 & ATCATTCATAAGAAGGACCCCAT & 157 & NA \\
\hline & & & & AGAAGTGGCAATATGGAATGTCT & & \\
\hline 26 & NAUPP_2569 & AG & 7 & TGATTTCAGCATAATAAATGCCC & 157 & ES \\
\hline & & & & ACAGAGAGCCAAAAGTAGCACAG & & \\
\hline 27 & NAUPP_2936 & $\mathrm{T}$ & 15 & AGATCTCCAACAAGAAAGAACCC & 141 & ES \\
\hline & & & & CCGGATTGTTCATCCAATATAAG & & \\
\hline 28 & NAUPP_2974 & CTA & 5 & TACCCGTTTTCTGAGTAACCAAA & 114 & ES \\
\hline & & & & GACGAAAATCGAACCTAAGACCT & & \\
\hline 29 & NAUPP_3021 & $\mathrm{AC}$ & 7 & CGAGTAATAAAGACGTCCAGGG & 160 & ES \\
\hline & & & & GCACTCCAAAAATCTAATTGTGC & & \\
\hline
\end{tabular}


Table 5. Continued.

\begin{tabular}{|c|c|c|c|c|c|c|}
\hline Code & Primer & $\begin{array}{l}\text { SSR } \\
\text { motif }\end{array}$ & $\begin{array}{c}\text { Number } \\
\text { of repeats }\end{array}$ & Primer sequence $\left(5^{\prime} \rightarrow 3^{\prime}\right)$ & $\begin{array}{l}\text { Except } \\
\text { size/bp }\end{array}$ & Product \\
\hline \multirow{2}{*}{30} & \multirow{2}{*}{ NAUPP_3106 } & \multirow{2}{*}{$\mathrm{CT}$} & \multirow{2}{*}{7} & ACTCGCAAAAATACACAAAAAGC & \multirow{2}{*}{133} & \multirow{2}{*}{ NA } \\
\hline & & & & CAACTCTCTCACТCTCTCСССТT & & \\
\hline \multirow[t]{2}{*}{31} & \multirow[t]{2}{*}{ NAUPP_3153 } & \multirow[t]{2}{*}{$\mathrm{AG}$} & \multirow[t]{2}{*}{9} & AAGAGCCATTGAGGAGCAATAG & \multirow[t]{2}{*}{139} & \multirow[t]{2}{*}{ ES } \\
\hline & & & & TTCTCTTCTATTTCTTTCGCCG & & \\
\hline \multirow[t]{2}{*}{32} & \multirow[t]{2}{*}{ NAUPP_3207 } & \multirow[t]{2}{*}{ CTC } & \multirow[t]{2}{*}{5} & CTTCCTAACTCCGACCAAAACTC & \multirow[t]{2}{*}{116} & \multirow[t]{2}{*}{ NA } \\
\hline & & & & CCTCAGAAGAAGCATCAACAAAC & & \\
\hline \multirow[t]{2}{*}{33} & \multirow[t]{2}{*}{ NAUPP_3297 } & \multirow[t]{2}{*}{ AGC } & \multirow[t]{2}{*}{5} & ATATGAACCGTAACGACGATCC & \multirow[t]{2}{*}{146} & \multirow[t]{2}{*}{-} \\
\hline & & & & GAACAGCTGCTCATACTTCATCC & & \\
\hline \multirow[t]{2}{*}{34} & \multirow{2}{*}{ NAUPP_3375 } & \multirow[t]{2}{*}{ TA } & \multirow[t]{2}{*}{9} & GAAGTGCGTGTAAAATCACACAA & \multirow{2}{*}{150} & \multirow[t]{2}{*}{ ES } \\
\hline & & & & CAAACCAACCCTAGCTACTCTCC & & \\
\hline \multirow[t]{2}{*}{35} & \multirow[t]{2}{*}{ NAUPP_3484 } & \multirow[t]{2}{*}{ CAC } & \multirow[t]{2}{*}{6} & CAAACACCAATTTCCTCAAAAAC & 160 & - \\
\hline & & & & GATCGATCTTGGATGTGGGT & & \\
\hline 36 & NAUPP_3502 & AG & 7 & TTGGTTTCTGAGACTTGCTTTGT & 120 & ES \\
\hline & & & & ACTTCTCCCTCCGTTGTTTAGTC & & \\
\hline 37 & NAUPP_3580 & $\mathrm{CT}$ & 6 & TTTAATTACAGCACTCGCCTCTC & 124 & ES \\
\hline & & & & GAAAATAACATGGGTTTTCTGGG & & \\
\hline$\overline{38}$ & NAUPP_3638 & $\mathrm{AG}$ & 6 & GTACTTGTTGATGCTTGTTGCAG & 138 & NA \\
\hline & & & & ATCATAGCAGTAATTCTGGCAGC & & \\
\hline 39 & NAUPP_3815 & G & 19 & AAGAGTGGGGGTTTGTATTGG & 147 & ES \\
\hline & & & & ACCCCGAATTAAGAGTTTTAGCA & & \\
\hline 40 & NAUPP_3982 & CT & 6 & ACACAAGGTTATTGATATGCGCT & 152 & ES \\
\hline & & & & CTCGACATCGATACAATTCAACA & & \\
\hline
\end{tabular}

$\mathrm{ES}=$ met expected size; $\mathrm{NA}=$ no amplification product; - = smaller than expected size .

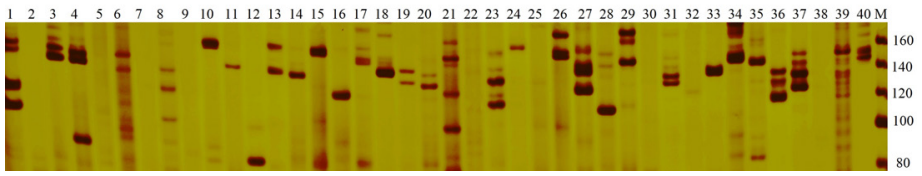

Figure 2. PAGE results from the PCR amplification of 40 primer pairs. Primer codes are the same as those listed in Table 5; Lane M: 20-bp DNA Ladder Marker (TaKaRa).

Table 6. COG classification of SSR-containing unigenes.

\begin{tabular}{|c|c|c|}
\hline Code & Functional categories of COG & Gene number \\
\hline $\mathrm{A}$ & RNA processing and modification & 52 \\
\hline $\mathrm{B}$ & Chromatin structure and dynamics & 54 \\
\hline $\mathrm{C}$ & Energy production and conversion & 103 \\
\hline $\mathrm{D}$ & Cell cycle control, cell division, chromosome partitioning & 152 \\
\hline E & Amino acid transport and metabolism & 152 \\
\hline $\mathrm{F}$ & Nucleotide transport and metabolism & 45 \\
\hline $\mathrm{G}$ & Carbohydrate transport and metabolism & 228 \\
\hline $\mathrm{H}$ & Coenzyme transport and metabolism & 59 \\
\hline $\mathrm{I}$ & Lipid transport and metabolism & 110 \\
\hline $\mathrm{J}$ & Translation, ribosomal structure, and biogenesis & 186 \\
\hline $\mathrm{K}$ & Transcription & 513 \\
\hline $\mathrm{L}$ & Replication, recombination, and repair & 338 \\
\hline $\mathrm{M}$ & Cell wall/membrane/envelope biogenesis & 112 \\
\hline $\mathrm{N}$ & Cell motility & 9 \\
\hline $\mathrm{O}$ & Posttranslational modification, protein turnover, chaperones & 250 \\
\hline$P$ & Inorganic ion transport and metabolism & 111 \\
\hline $\mathrm{Q}$ & Secondary metabolites biosynthesis, transport, and catabolism & 87 \\
\hline $\mathrm{R}$ & General function prediction only & 759 \\
\hline $\mathrm{S}$ & Function unknown & 142 \\
\hline $\mathrm{T}$ & Signal transduction mechanisms & 328 \\
\hline $\mathrm{U}$ & Intracellular trafficking, secretion, and vesicular transport & 66 \\
\hline $\mathrm{V}$ & Defense mechanisms & 31 \\
\hline $\mathrm{W}$ & Extracellular structures & 2 \\
\hline $\mathrm{Y}$ & Nuclear structure & 0 \\
\hline $\mathrm{Z}$ & Cytoskeleton & 35 \\
\hline
\end{tabular}

Genetics and Molecular Research 15 (3): gmr.15038683 


\section{DISCUSSION}

\section{Development and characterization of EST-SSRs in P. pyrifolia}

A total of 10,622 SSRs were obtained from 75,764 unigenes, with a frequency distribution of $1 / 5.24 \mathrm{~kb}$. The frequency distribution in the P. pyrifolia Nakai 'Huanghua' transcriptome was lower than that of P. bretschneideri Rehd. 'Dangshansu' (1/2.78kb) (Zhang et al., 2014). This finding could be due to differences between these two types of pear transcriptome, which may also be greatly affected by SSR-detecting criteria. In SSR development from the transcriptome, the first step was to define SSRs as a certain criterion, which decided the parameters of the SSR search algorithm, thereby directly affecting the final results. An exact comparison of SSR features between different species was complicated due to multiple factors. The frequency of pear EST-SSRs was higher than that of citrus $(1 / 5.7 \mathrm{~kb})$ (Chen et al., 2006), soybean $(1 / 23.8 \mathrm{~kb})$ (Gao et al., 2003), and barley (Hordeum vulgare L., 1/6.3 kb) (Thiel et al., 2003), but lower than kiwifruit (Actinidia spp, 1/2.48 kb) (Jiang et al., 2009) and tea (Camellia sinensis, 1/3.68 kb) (Yang et al., 2011). These differences could be the real cause of SSR frequency between different species, or it may be due to differences in the database used and SSR-detecting criteria. Overall, the SSRs of $P$. pyrifolia Nakai were plentiful.

Repeat motifs among these SSRs consisted of 159 types. A/T motifs occurred much more frequently than $\mathrm{C} / \mathrm{G}$ motifs, which is in agreement with studies of other plants (Morgante et al., 2002; Qiu et al., 2010; Guan et al., 2013). AG/CT was the most abundant motif and showed a striking dominance of all SSRs. The same results were reported in strawberry (Bombarely et al., 2010), lotus (Pan et al., 2010), and cassava (Raji et al., 2009). This is probably because the AG/CT dinucleotide motif can represent multiple codons resting within the reading frame that can be translated into different amino acids, and $\mathrm{AG} / \mathrm{CT}$ could be present in codons for alanine and leucine, which have the highest frequency in proteins. The frequency of the GC motif $(\mathrm{N}=11)$ was rare in our study, and similar results have been reported in previous studies. There were no GC motifs in Arabidopsis thaliana, apricot, peach, rice, maize, wheat, and soybean (Gao et al., 2003; Nicot et al., 2004; Jung et al., 2005), otherwise only two and one GC motifs were identified in coffee and kiwifruit, respectively (Aggarwal et al., 2007; Jiang et al., 2009). These reports have shown that plants may have an obvious GC bias, and the reasons for this phenomenon require further study. Among the trinucleotide motifs, AAG/CTT (662, 6.23\%) occurred most frequently, agreeing with results in coffee (Aggarwal et al., 2007), P. bretschneideri Rehd. (Zhang et al., 2014), Jatropha curcas L. (Yadav et al., 2011), and many other dicotyledonous plants (Kumpatla and Mukhopadhyay, 2005; Raji et al., 2009; Pan et al., 2010).

\section{Co-specific transferability and amplification rate}

Transcriptome sequencing has provided the unprecedented opportunity to mine ESTSSRs by generating massive amounts of available sequence data. Compared with DNA-derived SSRs, EST-SSRs have several obvious advantages. High co-specific transferability may be the most important, which has been demonstrated. Due to the source of EST-SSRs (directly derived from transcripts), they are more conservative and have greater transferability between species than genomic SSRs, which has been demonstrated in previous studies (Decroocq et al., 2003; Castillo et al., 2008; Shirasawa et al., 2011). These EST-SSRs, which have been mined from the pear transcriptome and are reported in our paper, surely can be used in other species

Genetics and Molecular Research 15 (3): gmr.15038683 
of Rosaceae, especially in those with limited molecular markers.

In total, 4300 primer pairs were obtained (Table S1), and 40 primer pairs were randomly selected for PCR amplification and PAGE analysis. Of these primers, 31 primer pairs $(77.50 \%)$ were successfully amplified. The success rate of amplification for these ESTSSRs was relatively high. The remaining 9 primers could not be amplified, because they might extend across a splice site or be derived from a chimeric cDNA sequence. Another reason might be that these primers were designed from questionable sequences based on transcriptome sequencing and would have inevitably failed when authenticated with genomic DNA. Among the 31 successfully amplified primer pairs, 29 were the expected size and 2 were smaller products than expected. Compared with DNA-derived SSRs, the amplification size of EST-SSRs tends to be more frequently derived from the expected size, which has been demonstrated in previous studies (Cordeiro et al., 2001; Thiel et al., 2003; Yu et al., 2004). This result might be due to introns or insertions-deletions within the amplicons. Moreover, the possibility of assembly errors, which occurred during transcriptome sequencing, cannot be excluded. In addition, the high success rate of amplification also confirmed that transcriptome sequencing is a fast, effective, and reliable approach to develop an abundance of available EST-SSRs.

\section{Level of potential polymorphism}

High polymorphism is one of the most important features of an effective SSR. Generally, perfect SSRs have higher polymorphisms than imperfect or compound SSRs (Weber, 1990). SSR polymorphisms are also affected by location within DNA. SSRs harbored within UTRs (untranslated regions) are more polymorphic than those occurring within exon regions (Qiu et al., 2010). This phenomenon might be caused by selection and evolution. SSRs harbored within UTRs would be easier to change than those within exon regions. Of the six types of SSR motifs, mononucleotide, dinucleotide, tetranucleotide, and pentanucleotide motifs mainly occurred within UTRs and had greater potential of being polymorphic. In contrast, trinucleotide and hexanucleotide repeat loci mainly occurred within exon regions and had lower potential of being polymorphic.

The length of SSRs is also an important factor regarding polymorphisms. Longer SSRs tend to be highly polymorphic and have a higher potential to serve as effective genetic markers, which have been shown in many organisms (Weber, 1990; Cho et al., 2000). According to the length of SSRs, Temnykh et al. (2001) categorized SSRs into two groups: Class I microsatellites, containing perfect SSRs $\geq 20$ nucleotides in length, and Class II microsatellites, containing perfect SSRs $>12$ and $<20$ nucleotides in length. Class I SSRs are deemed as having a high chance of showing a polymorphism.

In our report, a total of 10,622 SSRs were identified, of which $2293(21.59 \%)$ were $\geq 20$ nucleotides in length. These 2293 SSRs included mononucleotide (282), dinucleotide (1066), tetranucleotide (146), and pentanucleotide (216) motifs, which totaled 1710. These 1710 highly polymorphic SSRs, which were identified and designed in this study, will provide plenty of molecular markers for further genetic analyses of pears and other species in the Rosaceae.

\section{Annotation of SSR-containing unigenes}

Strong evidence supports the hypothesis that EST-SSRs play functional roles in organisms, some of which are regulatory (Li et al., 2004). In this study, functional annotations 
of SSR-containing sequences were reported, and a relatively higher percentage of unigenes (70.84\%) had BLAST hits in the Nr database, which was partially due to the high percentage frequency of long sequences in our assembled unigenes (nearly half of them were longer than 500 bp). As reported in some previous studies (Parchman et al., 2010; Wang et al., 2010b), longer assembled sequences have a higher probability of being annotated in public databases. EST-SSRs, which were mined from the transcriptome, have the potential to serve as functional markers, because they were directly derived from coding regions and some of them are linked with important candidate genes. Through these functional annotations, we can understand the distribution and function of SSR-containing unigenes at the macrolevel, and they are also very valuable for screening SSR markers, which are linked to functional genes.

In conclusion, our paper is the first to annotate pear genic SSR-containing sequences using public databases, GO terms, and COG categories. It is also one of the few studies that have developed EST-SSRs from the pear transcriptome. Pear EST-SSRs exhibited a relatively high frequency of SSR loci, which provide substantial resources for the development of ESTSSR markers. Considering self-incompatibility, high heterozygosity, and poorly differentiating characters of pears, these EST-SSRs should be particularly valuable for use in genomic mapping, breeding selection, phylogenetic relationship analysis, DNA fingerprinting, and other genetic studies. They can also be used in other relevant species because of their high co-specific transferability.

\section{Conflicts of interest}

The authors declare no conflict of interest.

\section{ACKNOWLEDGMENTS}

Research supported by the National Natural Science Foundation of China (\#31272140).

\section{REFERENCES}

Aggarwal RK, Hendre PS, Varshney RK, Bhat PR, et al. (2007). Identification, characterization and utilization of ESTderived genic microsatellite markers for genome analyses of coffee and related species. Theor. Appl. Genet. 114: 359-372. http://dx.doi.org/10.1007/s00122-006-0440-x.

Bombarely A, Merchante C, Csukasi F, Cruz-Rus E, et al. (2010). Generation and analysis of ESTs from strawberry (Fragaria xananassa) fruits and evaluation of their utility in genetic and molecular studies. BMC Genomics 11: 503. http://dx.doi.org/10.1186/1471-2164-11-503.

Castillo A, Budak H, Varshney RK, Dorado G, et al. (2008). Transferability and polymorphism of barley EST-SSR markers used for phylogenetic analysis in Hordeum chilense. BMC Plant Biol. 8: $97 \mathrm{http} / / \mathrm{dx}$. doi.org/10.1186/1471-2229-8-97.

Chen C, Zhou P, Choi YA, Huang S, et al. (2006). Mining and characterizing microsatellites from citrus ESTs. Theor. Appl. Genet. 112: 1248-1257 http://dx.doi.org/10.1007/s00122-006-0226-1.

Cho YG, Ishii T, Temnykh S, Chen X, et al. (2000). Diversity of microsatellites derived from genomic libraries and GenBank sequences in rice (Oryza sativa L.). Theor. Appl. Genet. 100: 713-722 http://dx.doi.org/10.1007/s001220051343.

Conesa A, Götz S, García-Gómez JM, Terol J, et al. (2005). Blast2GO: a universal tool for annotation, visualization and analysis in functional genomics research. Bioinformatics 21: 3674-3676. http://dx.doi.org/10.1093/bioinformatics/bti610.

Cordeiro GM, Casu R, McIntyre CL, Manners JM, et al. (2001). Microsatellite markers from sugarcane (Saccharum spp.) ESTs cross transferable to erianthus and sorghum. Plant Sci. 160: 1115-1123. http://dx.doi.org/10.1016/S01689452(01)00365-X.

Decroocq V, Fave MG, Hagen L, Bordenave L, et al. (2003). Development and transferability of apricot and grape EST microsatellite markers across taxa. Theor. Appl. Genet. 106: 912-922.

Genetics and Molecular Research 15 (3): gmr.15038683 
Fan L, Zhang MY, Liu QZ, Li LT, et al. (2013). Transferability of newly developed pear SSR markers to other Rosaceae species. Plant Mol. Biol. Report. 31: 1271-1282. http://dx.doi.org/10.1007/s11105-013-0586-z.

Gao L, Tang J, Li H and Jia J (2003). Analysis of microsatellites in major crops assessed by computational and experimental approaches. Mol. Breed. 12: 245-261. http://dx.doi.org/10.1023/A:1026346121217

Guan L, Huang JF, Feng GQ, Wang XW, et al. (2013). Survey of simple sequence repeats in woodland strawberry (Fragaria vesca). Genet. Mol. Res. 12: 2637-2651. http://dx.doi.org/10.4238/2013.July.30.3.

Jiang CY, Xu XB, Liao J, Ni ZH, et al. (2009). Analysis of SSR information in EST resources of kiwifruit (Actinidia ssp.). Zhongguo Nongxue Tongbao 13: 37-39.

Jung S, Abbott A, Jesudurai C, Tomkins J, et al. (2005). Frequency, type, distribution and annotation of simple sequence repeats in Rosaceae ESTs. Funct. Integr. Genomics 5: 136-143. http://dx.doi.org/10.1007/s10142-005-0139-0.

Kumpatla SP and Mukhopadhyay S (2005). Mining and survey of simple sequence repeats in expressed sequence tags of dicotyledonous species. Genome 48: 985-998. http://dx.doi.org/10.1139/g05-060.

Li YC, Korol AB, Fahima T and Nevo E (2004). Microsatellites within genes: structure, function, and evolution. Mol. Biol. Evol. 21: 991-1007. http://dx.doi.org/10.1093/molbev/msh073

Morgante M, Hanafey M and Powell W (2002). Microsatellites are preferentially associated with nonrepetitive DNA in plant genomes. Nat. Genet. 30: 194-200. http://dx.doi.org/10.1038/ng822.

Nicot N, Chiquet V, Gandon B, Amilhat L, et al. (2004). Study of simple sequence repeat (SSR) markers from wheat expressed sequence tags (ESTs). Theor. Appl. Genet. 109: 800-805. http://dx.doi.org/10.1007/s00122-004-1685-x

Pan H, Yang CP, Wei ZG and Jiang J (2006). DNA extraction of birch leaves by improved CTAB method and optimization of its ISSR system. J. For. Res. 17: 298-300. http://dx.doi.org/10.1007/s11676-006-0068-3.

Pan L, Xia Q, Quan Z, Liu H, et al. (2010). Development of novel EST-SSRs from sacred lotus (Nelumbo nucifera Gaertn) and their utilization for the genetic diversity analysis of $N$. nucifera. J. Hered. 101: 71-82. http://dx.doi.org/10.1093/ jhered/esp070

Parchman TL, Geist KS, Grahnen JA, Benkman CW, et al. (2010). Transcriptome sequencing in an ecologically important tree species: assembly, annotation, and discovery. BMC Genomics 11: 180. http://dx.doi.org/10.1186/1471-2164-11-180.

Potter D, Eriksson T, Evans RC, Oh S, et al. (2007). Phylogeny and classification of Rosaceae. Plant Syst. Evol. 266: 5-43. http://dx.doi.org/10.1007/s00606-007-0539-9.

Qiu L, Yang C, Tian B, Yang JB, et al. (2010). Exploiting EST databases for the development and characterization of EST-SSR markers in castor bean (Ricinus communis L.). BMC Plant Biol. 10: 278. http://dx.doi.org/10.1186/14712229-10-278.

Raji AA, Anderson JV, Kolade OA, Ugwu CD, et al. (2009). Gene-based microsatellites for cassava (Manihot esculenta Crantz): prevalence, polymorphisms, and cross-taxa utility. BMC Plant Biol. 9: 118. http://dx.doi.org/10.1186/14712229-9-118.

Shen ZJ, Ma RJ, Cai ZX, Yu ML, et al. (2015). Diversity, population structure, and evolution of local peach cultivars in China identified by simple sequence repeats. Genet. Mol. Res. 14: 101-117. http://dx.doi.org/10.4238/2015. January.15.13.

Shirasawa K, Oyama M, Hirakawa H, Sato S, et al. (2011). An EST-SSR linkage map of Raphanus sativus and comparative genomics of the Brassicaceae. DNA Res. 18: 221-232. http://dx.doi.org/10.1093/dnares/dsr013.

Tautz D and Renz M (1984). Simple sequences are ubiquitous repetitive components of eukaryotic genomes. Nucleic Acids Res. 12: 4127-4138. http://dx.doi.org/10.1093/nar/12.10.4127.

Temnykh S, DeClerck G, Lukashova A, Lipovich L, et al. (2001). Computational and experimental analysis of microsatellites in rice (Oryza sativa L.): frequency, length variation, transposon associations, and genetic marker potential. Genome Res. 11: 1441-1452. http://dx.doi.org/10.1101/gr.184001.

Thiel T, Michalek W, Varshney R and Graner A (2003). Exploiting EST databases for the development and characterization of gene-derived SSR-markers in barley (Hordeum vulgare L.). Theor. Appl. Genet. 106: 411-422.

Wang XC, Qiao YS, Wang F, Ma L, et al. (2010a). Extraction of total RNA from pericarp of pears with three methods. $J$. Gansu Agric. Univ. 45: 91-94.

Wang XW, Luan JB, Li JM, Bao YY, et al. (2010b). De novo characterization of a whitefly transcriptome and analysis of its gene expression during development. BMC Genomics 11: 400. http://dx.doi.org/10.1186/1471-2164-11-400.

Wang YW, Samuels TD and Wu YQ (2011). Development of 1,030 genomic SSR markers in switchgrass. Theor. Appl. Genet. 122: 677-686. http://dx.doi.org/10.1007/s00122-010-1477-4.

Weber JL (1990). Informativeness of human $(\mathrm{dC}-\mathrm{dA})_{\mathrm{n}} \cdot(\mathrm{dG}-\mathrm{dT})_{\mathrm{n}}$ polymorphisms. Genomics 7: 524-530. http://dx.doi. org $/ 10.1016 / 0888-7543(90) 90195-Z$

Wu J, Wang Z, Shi Z, Zhang S, et al. (2013). The genome of the pear (Pyrus bretschneideri Rehd.). Genome Res. 23: 396408. http://dx.doi.org/10.1101/gr.144311.112.

Genetics and Molecular Research 15 (3): gmr.15038683 
Yadav HK, Ranjan A, Asif MH, Mantri S, et al. (2011). EST-derived SSR markers in Jatropha curcas L.: development, characterization, polymorphism, and transferability across the species/genera. Tree Genet. Genomes 7: 207-219. http://dx.doi.org/10.1007/s11295-010-0326-6

Yang H, Chen Q, Wei CL, Shi CY, et al. (2011). Analysis on SSR information in Camellia sinensis transcriptome. J. Anhui Agric. Univ. 38: 882-886.

Ye J, Fang L, Zheng HK, Zhang Y, et al. (2006). WEGO: a web tool for plotting GO annotations. Nucleic Acids Res. 34: 293-297 http://dx.doi.org/10.1093/nar/gk1031.

Yu JK, Dake TM, Singh S, Benscher D, et al. (2004). Development and mapping of EST-derived simple sequence repeat markers for hexaploid wheat. Genome 47: 805-818. http://dx.doi.org/10.1139/g04-057

Yue XY, Liu GQ, Zong Y, Teng YW, et al. (2014). Development of genic SSR markers from transcriptome sequencing of pear buds. J. Zhejiang Univ. Sci. 15: 303-312. http://dx.doi.org/10.1631/jzus.B1300240.

Zhang MY, Fan L, Liu QZ, Song Y, et al. (2014). A novel set of EST-derived SSR markers for pear and cross-species transferability in Rosaceae. Plant Mol. Biol. Report. 32: 290-302. http://dx.doi.org/10.1007/s11105-013-0638-4.

\section{Supplementary material}

Table S1. Primer results after filtering (4300 pairs of primers). 